\title{
Action plan for improving cardiac rehabilitation-related outcomes in a university hospital based on a review of previous interventions
}

\author{
Carol Elsakr ${ }^{1}$, Leesa Wright ${ }^{2}$, Pooja S. Jagadish ${ }^{3}$, David A. Bulger ${ }^{3}$, Uzoma N. Ibebuogu ${ }^{4}$, Rami N. Khouzam $^{4}$ \\ ${ }^{1}$ Department of Medicine, The George Washington University School of Medicine \& Health Sciences, Washington, DC, USA; ${ }^{2}$ Methodist \\ University Hospital, Memphis, TN, USA; ${ }^{3}$ Department of Internal Medicine, ${ }^{4}$ Division of Cardiovascular Diseases, Department of Internal \\ Medicine, University of Tennessee Health Science Center, Memphis, TN, USA \\ Contributions: (I) Conception and design: C Elsakr, PS Jagadish, RN Khouzam, L Wright; (II) Administrative support: RN Khouzam, L Wright, UN \\ Ibebuogu; (III) Provision of study materials or patients: L Wright; (IV) Collection and assembly of data: C Elsakr, L Wright; (V) Data analysis and \\ interpretation: C Elsakr, L Wright, DA Bulger; (VI) Manuscript writing: All authors; (VII) Final approval of manuscript: All authors. \\ Correspondence to: Carol Elsakr. BS. 2300 I St. NW, Washington, DC 20052, USA. Email: celsakr@gwu.edu.
}

\begin{abstract}
Although referral to cardiac rehabilitation (CR) is considered the standard of care and demonstrably reduces both mortality rates and hospital admissions after cardiac events, rates of referral continue to be suboptimal. In fact, national reports reveal rates ranging from approximately $60 \%$ to $85 \%$ depending on the type of cardiac event. At an urban teaching hospital in Tennessee, efforts to increase referral rates were launched during the first quarter of 2018 as part of the Define, Measure, Analyze, Improve, Control (DMAIC) Project: Acute Myocardial Infarction (AMI) Transition of Care. The goal of this Action Plan is to review the interventions taken and the outcomes data from this project in order to propose future deliverables that can address areas of improvement within the DMAIC project. A list of the DMAIC project's interventions, which were varied and multidisciplinary, were obtained from the university hospital as well as the project's data. Data from the National Cardiovascular Data Registry (NCDR)-ACTION Registry show that referral rates at this hospital have been on the rise since the initiation of the DMAIC project. Peak referral rates in the year before the interventions were implemented were approximately $39 \%$; whereas, the peak referral rate in the year these interventions were launched rose to $86.4 \%$. While the interventions of the DMAIC project are hypothesized to have contributed to this increase in referral rates, based on their collaborative nature and the types of referral strategies employed, there are still opportunities for improvement and growth. Thus, this Action Plan proposes future projects to increase inclusivity of CR referral pathways, improve physician education, and establish support for outpatient CR programs.
\end{abstract}

Keywords: Cardiac rehabilitation (CR); consultation; myocardial infarction; quality improvement; referral

Submitted Jun 30, 2019. Accepted for publication Jul 25, 2019.

doi: 10.21037/atm.2019.08.09

View this article at: http://dx.doi.org/10.21037/atm.2019.08.09

\section{Introduction}

Cardiac rehabilitation (CR) exists as an exercise-based, educational, and often multi-modal secondary prevention program for patients with cardiovascular disease (1), and it demonstrably reduces mortality rates $(2,3)$ and hospital admissions (4,5). The 2010 American Association of Cardiovascular and Pulmonary Rehabilitation/ American College of Cardiology Foundation/American
Heart Association (AACVPR/ACCF/AHA) update on performance measures on CR favors referral to CR among a broad-based patient population (6). This includes those with a recent coronary artery bypass graft (CABG), revascularization procedure, or acute coronary syndrome (ACS) event as well as those with heart failure (Class I, Level of Evidence B for all populations) (6).

Methodist University Hospital (MUH) in Memphis, TN 
Table 1 Summary of various interventions of the DMAIC project, which began development and implementation in early 2018. Information provided by MUH

\author{
Interventions of the DMAIC project of 2018 \\ Development of an improved documentation tool for nurses \\ Development of a shared documentation tool between case management and other hospital staff \\ Education of non-cardiology admitting physicians regarding ACS order sets \\ Cardiology quality improvement fellow tasked with improving compliance with ACS order set utilization \\ Collaboration between the chief of cardiology and quality improvement personnel (e.g., creating performance indicators around \\ compliance with ACS order set utilization) \\ Routine inpatient phase I CR team's evaluations of patients for CR referral \\ Education of case management and abstractors on CR referral processes and documentation \\ Instruction of abstractors to provide feedback on patients whose care has yet to meet all standard care requirements before discharge \\ New designs of visual data related to quality measurements for the purpose of improving their comprehensibility to various hospital staff \\ Recruitment of support from hospital administration for the DMAIC project
}

DMAIC, Define, Measure, Analyze, Improve, Control; ACS, acute coronary syndromes; CR, cardiac rehabilitation; MUH, Methodist University Hospital.

has developed and implemented multiple interventions for increasing the number of appropriate referrals to CR. This Action Plan reviews these interventions and their impact on referral rates. Based on these changes, further interventions are proposed.

\section{Methods}

MUH has key quality improvement personnel; one of their goals is to improve referral rates to CR post-ACS events. The Define, Measure, Analyze, Improve, Control (DMAIC) Project: Acute Myocardial Infarction (AMI) Transition of Care was the latest effort by MUH to improve CR referral rates. All data used in this paper was from the MUH DMAIC project database.

In the first quarter of 2018, the project initiated the early phases of its interventions. The interventions that this project included are listed in Table 1. First, the project called for the development of an improved documentation tool that allows nurses discharging CR-eligible patients to see the details of a patient's referral. Next, a shared documentation tool to facilitate communication between case management and other hospital staff was development and initiated. With those tasks in motion, education to non-cardiology admitting physicians (e.g., rotating residents) about CR and the ACS order set that prompts CR referral was prioritized.
A cardiology fellow was additionally tasked with improving compliance with order set utilization, and the chief of cardiology began working with a hospitalbased quality improvement team to create performance indicators to assess compliance with use.

As for improving the referral process itself, the inpatient phase I CR team's evaluation and education of patients was established as a crucial element to the referral. Case managers and abstractors were also educated about the CR referral process and the necessary documentation surrounding it. Abstractors were further instructed to provide feedback to prescribers on patients whose care did not yet meet all standard care requirements prior to discharge.

In order to promote a culture encouraging compliance with CR referrals, visual data related to the measurements of quality markers was redesigned so that hospital staff could best understand the importance of their tasks. Finally, the project team recruited support from hospital administration for the DMAIC project by providing information regarding the benefits of CR referrals.

The number of referrals to CR out of total patients admitted for AMI from 2017 to 2018 were tracked quarterly via abstractors, whose data were vetted by the National Cardiovascular Data Registry (NCDR)-ACTION Registry. Bar plot of data in Figure 1 was made using R Statistical Programming Language Version 3.6.0 (7). 


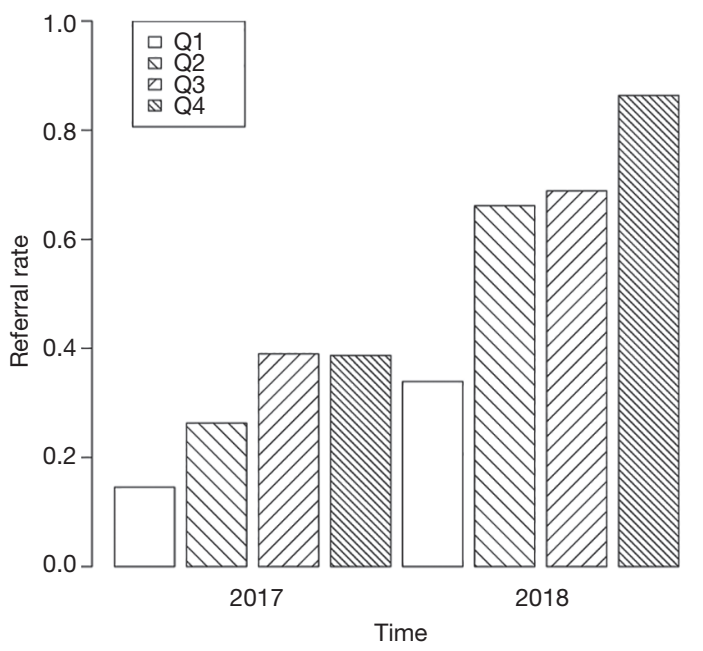

Figure 1 Referral rates of AMI patients to CR calculated for each quarter from 2017-2018. All data from MUH DMAIC project. AMI, acute myocardial infarction; CR, cardiac rehabilitation; MUH, Methodist University Hospital; DMAIC, Define, Measure, Analyze, Improve, Control.

Table 2 Referral data. Number of patients each quarter from 20172018 referred to CR out of number of patients admitted to MUH for AMI from the NCDR-ACTION Registry. All data from MUH DMAIC project (quarterly CR referral rates from 2017-2018)

\begin{tabular}{lccc}
\hline Year & Quarter & Numerator (referral) & Denominator (AMIs) \\
\hline 2017 & 1 & 8 & 55 \\
2017 & 2 & 15 & 57 \\
2017 & 3 & 23 & 59 \\
2017 & 4 & 24 & 62 \\
2018 & 1 & 19 & 56 \\
2018 & 2 & 41 & 62 \\
2018 & 3 & 42 & 61 \\
2018 & 4 & 38 & 44 \\
\hline
\end{tabular}

AMIs, acute myocardial infarctions; DMAIC, Define, Measure, Analyze, Improve, Control; CR, cardiac rehabilitation; $\mathrm{MUH}$, Methodist University Hospital; NCDR, National Cardiovascular Data Registry.

\section{Results}

Data from the NCDR-ACTION Registry on CR referrals from 2017-2018 are displayed in Table 2. During the first quarter (Q1) of 2017 CR referral rate was $14.5 \%(n=55)$. This rate gradually increased throughout 2017 with Q2 referral rate at $26.3 \%(\mathrm{n}=57)$ and Q3 referral rate at 39.0\% $(\mathrm{n}=59)$. However, there was a slight decline in the fourth quarter (Q4) of 2017 with referral rate at 38.7\% $(n=62)$. The next year referral rate continued to decline in the first quarter, but were still more than double the previous year with 2018 Q1 referral rate at 33.9\% $(n=56)$. The next three quarters of 2018 saw a continued increase in referral rates especially in comparison with the same quarter of the previous year: Q2 had rates 2.5 times higher at $66.1 \%$ $(\mathrm{n}=62), \mathrm{Q} 3 \mathrm{had}$ rates 1.8 times higher at $68.9 \%(\mathrm{n}=61)$, and Q4 had rates 2.2 times higher at $86.4 \%(n=44)$. The calculated referral rates are displayed in Figure 1.

\section{Discussion}

The above interventions have been successful at MUH with an increase in CR referral rate from $14.5 \%$ in the first quarter of 2017 to $86.4 \%$ in the fourth quarter of 2018 (Figure 1). This is a notable increase in the referral rate, especially when compared with national rates of referral. For example, in 2014, the ACTION-Get With the Guidelines registry showed $84.5 \%$ of ST-elevation myocardial infarction (STEMI) patients and $75.9 \%$ of NSTEMI patients were referred to CR (8). From 2009 to 2012, data from the NCDR showed around $60 \%$ of postpercutaneous coronary intervention (PCI) patients were referred (9). This recent increase in referral rates is likely due to the multidisciplinary targets and collaborative nature of the interventions by the DMAIC project.

Previous projects at $\mathrm{MUH}$ targeting referrals to $\mathrm{CR}$ were more exclusive in their interventions. For example, past projects focused solely on case management or solely on the inpatient CR team, rather than targeting both groups or promoting better communication between the groups and other hospital staff. With CR-referral rates as low as $14.5 \%$ in early 2017, it became clear that an interdisciplinary approach to this problem would be necessary.

\section{Referral strategies}

The interventions employed by the DMAIC project are also advantageous for referral rates and even other outcomes since they entail both automatic referrals and liaison referrals. In their review article, Gallagher and colleagues suggest that the "liaison type of referral strategy" is more educational for patients and could allow clinicians the opportunity to strongly recommend CR (10). This, in 
turn, could lead to an increase in participation rates after referral (10). Gravely-Witte et al. found in their review that studies examining automatic referrals achieved the highest rates of referral; studies examining a combination of liaison referral and automatic referral achieved the highest rates of enrollment (11).

The automatic referral strategy at MUH consists of nonphysician staff (e.g., case managers, CR team members, etc.) verbally ordering referrals to CR by default if the physician has not done so via the ACS order set. It should be noted that this form of automatic referral is considered the last resort for ensuring a quality standard-of-care and highlights how order set utilization is an area that continues to require improvement. The liaison referral strategy at MUH consists of inpatient CR team members evaluating and educating patients regarding CR referral.

\section{The future of the DMAIC project: interventions for improvement}

Despite the overall strength of the interventions employed in 2018, various parts of the project are still underway. There are also multiple areas where additional interventions could be employed to further optimize patient referral to CR as well as improve outcomes at the outpatient CR level.

First, while the project discussed in this Action Plan entailed efforts to create a CR pathway for patients with AMI, there are other categories of patients who should be offered CR per guidelines, such as those with heart failure (6). A CR pathway for such groups of patients has yet to be well-developed by MUH.

Second, great strides remain in the education of physicians on the benefits and referral process of CR. Gallagher and colleagues show concern that physicians attend potentially non-accredited online CR-courses due to the lack of a CR-specific international governing body or a paucity of CR-specialized graduate programs (10). Thus, the creation of an accredited, online CR course could satisfy this void and fill this need. A quarterly workshop as well as the inclusion of CR-referral training in the orientation of residents, fellows, and new physicians entering the hospital system could also be helpful in educating physicians at MUH. Alternatively, noon conferences, quarterly meetings, or continuing medical education (CME) events could be used for physician education. However, these interventions come at the cost of time and patience, and knowledge may not be optimally retained. It remains important to find the optimal means of physician education.

Next, the literature has revealed and commented on the apparent drop-off that can occur between referral and subsequent enrollment to CR (12-15). While CR referrals theoretically lead to enrollment, it is important to assess the actual enrollment and participation rates at those CR programs to which that MUH refers. This data could be used to create specific interventions that will concretely affect patient mortality and hospitalization rates rather than simply treating low CR-referral numbers with no patientrelated benefit.

Last, Grace and colleagues found that Canadian outpatient CR programs were unexpectedly strained due to the influx of patients after implementing successful referral strategies (16). It is very possible that the outpatient CR programs to which MUH refers are experiencing a similar burden. Assessing enrollment rates and current capacities of these programs would provide case managers with data on wait times for enrollment. This, in turn, would trigger referrals to less-overburdened programs, thus improving delays in care.

In order to optimally address the current areas for improvement in the DMAIC project, the following deliverables have been proposed:

(I) Creation of a preliminary protocol for referring nonAMI patients (e.g., those with heart failure) to CR;

(II) Development of an online CR course, accredited via the University of Tennessee Health Science Center;

(III) Improvement of the curriculum for hospital-based physician education to include CR;

(IV) Preparation of educational materials for CRtraining during orientations for residency and fellowship or during educational conferences;

(V) Tabulation of the enrollment rates, participation rates, capacities, and measures of resource strain at local outpatient CR programs.

These interventions are anticipated to allow more patients with different cardiovascular conditions to partake in a program that would be beneficial to them; they also provide an opportunity for closer relationships between hospitals and outpatient CR programs, which would also benefit patients' care.

\section{Conclusions}

In order to optimally improve the quality of care for 
cardiac patients at MUH, the DMAIC project set out to improve CR-referral rates with resounding success. This was evidenced by referral rates skyrocketing past current national averages. Yet, there are still many key areas for further improvement, from inclusion of heart failure patients in the CR-referral model to increased utilization of the ACS order set. In medicine, there is sometimes a tendency to "treat numbers," and creating tangible ways of measuring patient-based outcomes (e.g., mortality and hospitalization rates) are paramount to ensure that goals are truly being met. In other words, there should not only be a focus on CR-referral rates, but rates of CR-enrollment and participation must be evaluated as well. Through hospital team-member education and collaboration with community-based programs, the outcomes of cardiac patients can be tangibly improved. The deliverables described in this Action Plan are stepping stones in the pathway to ultimately achieving this end.

\section{Acknowledgments}

We would like to acknowledge Methodist University Hospital for the encouragement to undertake this research and create an action plan that other institutions may emulate. We would also like to acknowledge all those involved in making the DMAIC project a reality and their commitment to improving patient outcomes.

\section{Footnote}

Conflicts of Interest: The authors have no conflicts of interest to declare.

Ethical Statement: The authors are accountable for all aspects of the work in ensuring that questions related to the accuracy or integrity of any part of the work are appropriately investigated and resolved.

\section{References}

1. Freeman AM, Taub PR, Lo HC, et al. Intensive Cardiac Rehabilitation: an Underutilized Resource. Curr Cardiol Rep 2019;21:19.

2. Heran BS, Chen JM, Ebrahim S, et al. Exercise-based cardiac rehabilitation for coronary heart disease. Cochrane Database Syst Rev 2011;(7):CD001800.

3. Lawler PR, Filion KB, Eisenberg MJ. Efficacy of exercise-based cardiac rehabilitation postmyocardial infarction: a systematic review and metaanalysis of randomized controlled trials. Am Heart J 2011;162:571-84.e2.

4. Anderson L, Oldridge N, Thompson DR, et al. ExerciseBased Cardiac Rehabilitation for Coronary Heart Disease: Cochrane Systematic Review and Meta-Analysis. J Am Coll Cardiol 2016;67:1-12.

5. Sagar VA, Davies EJ, Briscoe S, et al. Exercise-based rehabilitation for heart failure: systematic review and meta-analysis. Open Heart 2015;2:e000163.

6. Thomas RJ, King M, Lui K, et al. AACVPR/ACCF/ AHA 2010 update: performance measures on cardiac rehabilitation for referral to cardiac rehabilitation/ secondary prevention services: a report of the American Association of Cardiovascular and Pulmonary Rehabilitation and the American College of Cardiology Foundation/American Heart Association Task Force on Performance Measures (Writing Committee to Develop Clinical Performance Measures for Cardiac Rehabilitation). Circulation 2010;122:1342-50.

7. R Core Team. R: A Language and Environment for Statistical Computing. R Foundation for Statistical Computing. Vienna, Austria: 2019.

8. Benjamin EJ, Blaha MJ, Chiuve SE, et al. Heart Disease and Stroke Statistics-2017 Update: A Report From the American Heart Association. Circulation 2017;135:e146-603.

9. Aragam KG, Dai D, Neely ML, et al. Gaps in referral to cardiac rehabilitation of patients undergoing percutaneous coronary intervention in the United States. J Am Coll Cardiol 2015;65:2079-88.

10. Gallagher R, Neubeck L, Du H, et al. Facilitating or getting in the way? The effect of clinicians' knowledge, values and beliefs on referral and participation. Eur J Prev Cardiol 2016;23:1141-50.

11. Gravely-Witte S, Leung YW, Nariani R, et al. Effects of cardiac rehabilitation referral strategies on referral and enrollment rates. Nat Rev Cardiol 2010;7:87-96.

12. Boyden T, Rubenfire M, Franklin B. Will increasing referral to cardiac rehabilitation improve participation? Prev Cardiol 2010;13:192-201.

13. Scott LB. Referral to outpatient cardiac rehabilitation: intervention research at the patient, provider, and health system levels. Nat Clin Pract Cardiovasc Med 2008;5:671-2.

14. Turk-Adawi KI, Oldridge NB, Tarima SS, et al. Cardiac 
rehabilitation enrollment among referred patients: patient and organizational factors. J Cardiopulm Rehabil Prev 2014;34:114-22.

15. Weingarten MN, Salz KA, Thomas RJ, et al. Rates of enrollment for men and women referred to outpatient cardiac rehabilitation. J Cardiopulm Rehabil Prev
2011;31:217-22.

16. Grace SL, Tan Y, Simpson C, et al. Perceptions of cardiac specialists and rehabilitation programs regarding patient access to cardiac rehabilitation and referral strategies. J Cardiopulm Rehabil Prev 2012;32:135-40.

Cite this article as: Elsakr C, Wright L, Jagadish PS, Bulger DA, Ibebuogu UN, Khouzam RN. Action plan for improving cardiac rehabilitation-related outcomes in a university hospital based on a review of previous interventions. Ann Transl Med 2019;7(17):415. doi: 10.21037/atm.2019.08.09 